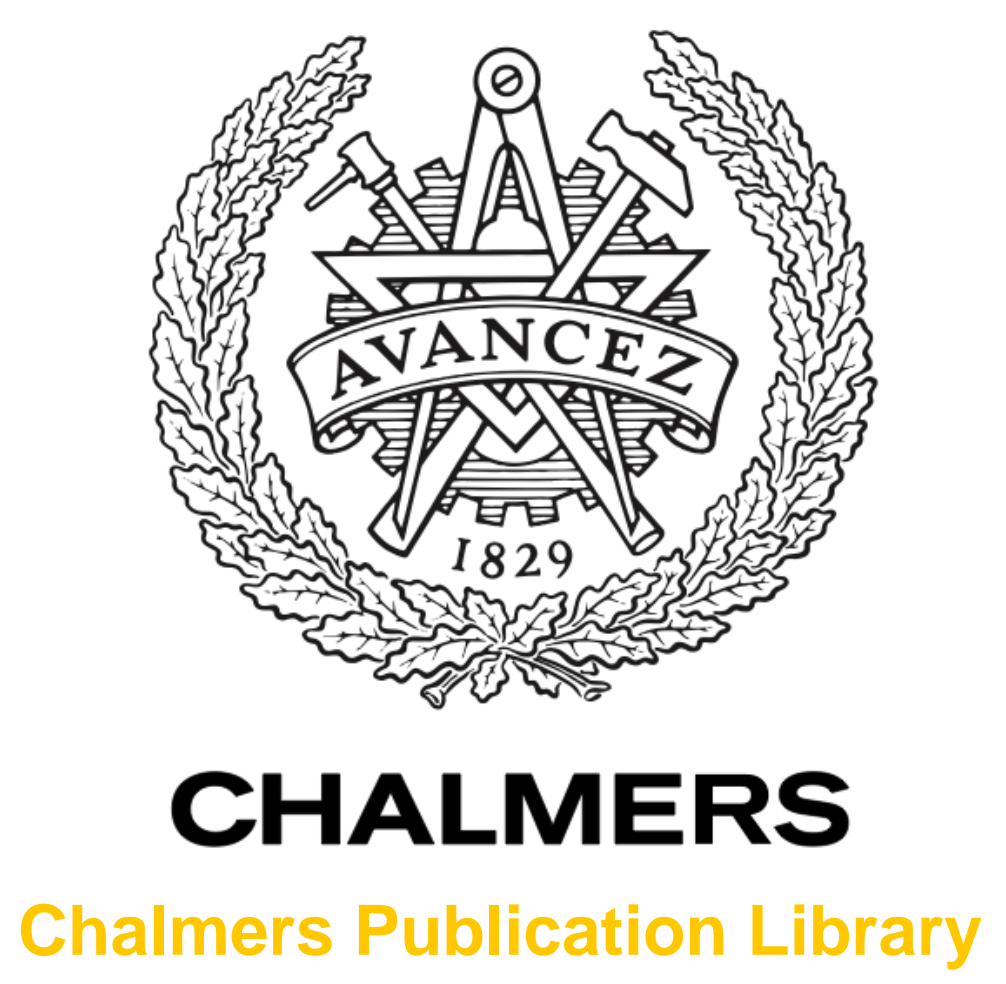

\title{
Orthotropic criteria for transverse failure of non-crimp fabric-reinforced composites
}

This document has been downloaded from Chalmers Publication Library (CPL). It is the author's version of a work that was accepted for publication in:

Journal of composite materials (ISSN: 0021-9983)

Citation for the published paper:

Molker, H. ; Wilhelmsson, D. ; Gutkin, R. et al. (2015) "Orthotropic criteria for transverse failure of non-crimp fabric-reinforced composites". Journal of composite materials

http://dx.doi.org/10.1177/0021998315605877

Downloaded from: http://publications.lib.chalmers.se/publication/223484

Notice: Changes introduced as a result of publishing processes such as copy-editing and formatting may not be reflected in this document. For a definitive version of this work, please refer to the published source. Please note that access to the published version might require a subscription.

Chalmers Publication Library (CPL) offers the possibility of retrieving research publications produced at Chalmers University of Technology. It covers all types of publications: articles, dissertations, licentiate theses, masters theses, conference papers, reports etc. Since 2006 it is the official tool for Chalmers official publication statistics. To ensure that Chalmers research results are disseminated as widely as possible, an Open Access Policy has been adopted.

The CPL service is administrated and maintained by Chalmers Library. 


\title{
Orthotropic criteria for transverse failure of non-crimp fabric reinforced composites
}

\section{Henrik Molker}

Durability CAE Body \& Trim, Volvo Car Corporation, 405 3I Gothenburg, Sweden

Department of Applied Mechanics, Chalmers University of Technology, 41296 Gothenburg, Sweden

\section{Dennis Wilhelmsson}

Department of Applied Mechanics, Chalmers University of Technology, 4I 296 Gothenburg, Sweden

\section{Renaud Gutkin}

Swerea SICOMP AB, Box 104, 43122 Mölndal, Sweden

\section{Leif E Asp}

Department of Applied Mechanics, Chalmers University of Technology, 41296 Gothenburg, Sweden

\begin{abstract}
In this paper, a set of failure criteria for transverse failure in Non Crimp Fabric (NCF) reinforced composites are presented. The proposed failure criteria are physically based and take into account the orthotropic character of NCF composites addressing the observed lack of transverse isotropy. Experimental data for transverse loading out of plane in combination with inplane loads are scarce. Therefore, to validate the developed criteria, experimental data are complemented with numerical data from a representative volume element (RVE) model using a meso-micromechanical approach. The RVE model also provides a deeper understanding of how failure occurs in NCF composites. Strength predictions from the developed set of failure criteria show good agreement with the experimental and numerical data.
\end{abstract}

\section{Corresponding author:}

Henrik Molker, Durability CAE Body \& Trim, Volvo Car Corporation, 405 3I, Gothenburg, Sweden henrik.molker@volvocars.com 


\section{Keywords}

Carbon fibre composites; Textile fabrics; Transverse strength; Failure envelopes; Representative Volume

Element

\section{Introduction}

When composites are introduced in applications where manufacturing cost and cycle time are important factors the need for rational and automated production methods becomes ever more important. The traditional way of making carbon fibre composites, by stacking a number of pre-impregnated sheets using inexpensive tooling and curing in an autoclave, is a labour intensive and time consuming process. Cost effective textile preforming techniques together with improved infusion techniques are promising methods [1] to overcome these obstacles. One important class of such composites are Non-Crimp Fabric composites (NCF).

Development of structures within aerospace and automotive industries today is driven using Computer Aided Engineering (CAE) tools in a virtual environment. To have an efficient design process for all parts in a system, CAE is needed also for Carbon Fibre Reinforced Polymer (CFRP) Composites. Robust and efficient design processes need accurate prediction of failure initiation and propagation up to final failure for static as well as dynamic loading events. Today, a generic methodology for analysis of automotive 
applications from CFRP is lacking. For full-scale analyses in automotive design, the methodology to be developed most likely needs to consider local-global analysis schemes as done in aircraft design today [2].

A common approach to analyse strength of laminated composites is to calculate a failure index for each ply within the laminate, and assume that failure occurs when the failure index in any of the plies reaches unity. This is referred to as "first ply failure". A wide range of failure criteria are currently available for traditional Uni-Directional (UD) composites, like tape-based (prepreg) systems. These include polynomial fits as the TsaiWu criterion [3] and physically based criteria e.g. the Puck [4] or LaRC05 [5] criteria. However, models need further development or refinement to be usable for new types of composites with textile reinforcement, e.g. NCF reinforced composites. Once such failure criteria are established for NCF reinforced composites they can be employed for hot-spot analyses. As such they will provide a very powerful framework for the analysis of structural components in large assemblies [2].

NCF reinforced composites have a different architecture on the meso-scale compared to prepregs. Due to the complex material architecture of textile reinforced composites 
numerical Finite Element (FE) models have predominately been used for their analysis [6]. In particular, such models are useful to predict strength of composite structures exposed to three-dimensional stress states. Furthermore, these numerical models contribute to a deeper understanding of the competing failure mechanisms that occur in textile composites. Examples of successful use of numerical multi-scale models for UDmaterials with transversely isotropic properties can also be found, e.g. see work by Camanho et al. [7].

In this paper, we present failure criteria for matrix dominated failure in NCF-reinforced composites. NCF-reinforced composites are not transversely isotropic materials. The proposed failure criteria are based on the physically based LaRC05 criteria and modified to take the orthotropic properties of the NCF composites into account. The criteria predict failure at ply level and uses data for homogenised ply properties. To validate the criteria, numerical experiments were performed to complement available experimental data from literature. In particular, numerical experiments were focused on combined in and out of plane load cases, for which there is a general lack of physical data. Numerical experiments were performed with a meso-mechanical FE-model considering a Representative Volume Element (RVE). The meso-mechanical model uses appropriate criteria for each 
constituent, in this case the impregnated fibre bundle and the surrounding matrix material. The numerical model also provides a tool for improved understanding of prevalent failure mechanisms in NCF reinforced composites exposed to complex 3D stress states.

\section{Material description}

Textile composites which consist of fibre tows with UD fibres kept together with a knitting yarn are known as NCF reinforced composites, see Figure 1(a). The advantage of these textiles compared to traditionally woven fabrics is that the waviness of the impregnated fibre tows is reduced. This improves the mechanical properties, especially in compression. The architecture of NCF textiles can be tailored to fit the needs of the structure. NCF blankets can consist of either a single layer of fibre tows in one direction, called an NCF uni-weave, or several fibre tow layers stacked in different directions and stitched together, i.e. a multi-axial NCF blanket. The layers are kept in place by knitting yarn in the warp (longitudinal) and weft (transverse) directions as illustrated in Figure 1(a) [8]. 


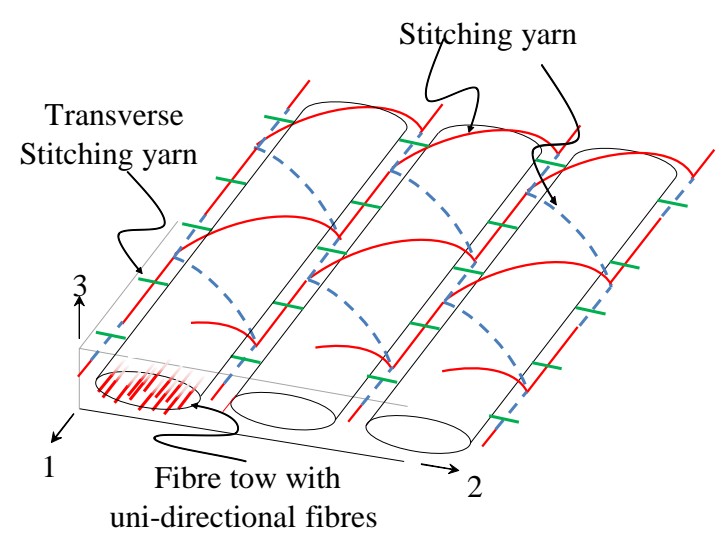

(a)

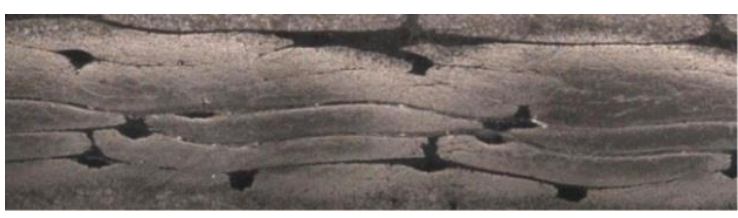

(b)

Figure I. (a) Schematic illustration of a NCF uni-weave with tricot knitting. (b) Cross section of NCF perpendicular to fibre bundles (from [6]). The shape of the bundles is distorted with irregular shape and interface to the matrix. Dark areas are resin pockets.

\section{The orthotropic nature of NCF reinforced composites}

The material architecture of an NCF reinforced composite, with fibre tows and matrix that are not symmetrically spaced, indicates that the behaviour is not isotropic, see Figure 1(b). Moreover, the 2-3 plane transverse to the fibre direction in Figure 1(a) is also orthotropic due to the knitting yarn that is used to keep the fibre tows and plies together within the blanket. When loaded in out of plane tension, a stress concentration is formed at the stitching yarn, which can cause a fracture in the 1-2 plane [9]. 
Irregularities in the shape of the fibre bundles, i.e. the impregnated fibre tows, cause stress concentrations at the interface between the fibre bundle and matrix in the 2-3 plane, i.e. the transverse plane. These stress concentrations may further reduce the strength of the composite. The distance between the fibre bundles is significantly smaller in the out of plane direction than in the plane and consequently stress and strain gradients will be greater for out of plane loads. The knitting yarn also affects the mechanical properties. In the in-plane direction the weft knitting yarns have the possibility to carry tensile loads as these fibers are stretched.

The stiffness and strength of NCF reinforced composites have been found to be non transversely isotropic in the 2-3 plane, see Figure 1(a). In contrast to UD composites the stiffness and strength values are different in the 2 and 3 directions [9]. Strength properties for a homogenised NCF reinforced composite and its constituents, i.e. matrix and fibre bundle, are listed in Table 1. In Table 2, elastic properties are listed for a homogenised NCF reinforced composite and its constituents.

Table I: Strength properties for the NCF reinforced composite and its constituents in the transverse plane.

\begin{tabular}{cccccc}
\hline & $\begin{array}{c}Y_{\mathrm{C}} \\
(\mathrm{MPa})\end{array}$ & $\begin{array}{c}Y_{\mathrm{T}} \\
(\mathrm{MPa})\end{array}$ & $\begin{array}{c}Z_{\mathrm{C}} \\
(\mathrm{MPa})\end{array}$ & $\begin{array}{c}Z_{\mathrm{T}} \\
(\mathrm{MPa})\end{array}$ & $\begin{array}{c}S_{\mathrm{L}} \\
(\mathrm{MPa})\end{array}$ \\
\hline NCF reinforced & 200 & 40 & 220 & 25 & 75 \\
composite [9] & & $($ std.dev $5 \%)$ & & $($ std.dev. 6\%) & 75 \\
Fibre Bundle [6] & $200^{\mathrm{a})}$ & 45 & - & - & -
\end{tabular}


a) Predicted value from [6].

b) The yield limit in compression is used as a strength value.

Table 2: Elastic properties of the NCF reinforced composite and its constituents.

\begin{tabular}{lcccccccc}
\hline & $\begin{array}{c}E_{11} \\
(\mathrm{GPa})\end{array}$ & $\begin{array}{c}E_{22} \\
(\mathrm{GPa})\end{array}$ & $v_{12}$ & $v_{23}$ & $\begin{array}{c}G_{23} \\
(\mathrm{GPa})\end{array}$ & $\begin{array}{c}\alpha_{0} \\
(\mathrm{deg})\end{array}$ & $\begin{array}{c}\alpha_{11} \\
\left(\mu \mathrm{m} / \mathrm{m}^{\circ} \mathrm{C}\right)\end{array}$ & $\begin{array}{c}\alpha_{22} \\
\left(\mu \mathrm{m} / \mathrm{m}^{\circ} \mathrm{C}\right)\end{array}$ \\
\hline $\begin{array}{l}\text { NCF reinforced } \\
\text { composite [9] }\end{array}$ & - & - & - & - & 4.2 & 55 & - & - \\
HTS Fibre [6] & 233 & 23.0 & 0.20 & 0.20 & - & - & -0.50 & 10.0 \\
Fibre Bundle [6] & 162 & 10.6 & 0.26 & $0.40^{\mathrm{a})}$ & 4.1 & - & -0.05 & 39.0 \\
RTM6 Matrix [6] & 3.10 & 3.10 & 0.38 & 0.38 & 1.1 & - & $70.0^{\mathrm{a})}$ & $70.0^{\mathrm{b})}$
\end{tabular}

a) Assumed value.

b) Thermal expansion coefficient is temperature dependent.

The tensile strength in the 3-direction, $Z_{T}$, has been found to be close to half of that in the 2-direction, $Y_{T}$, see Table 1 . In compression the difference is smaller and reversed, the out of plane compressive strength is approximately $10 \%$ lower in the 2-direction, $Y_{C}$, compared to the 3 -direction, $Z_{C}[9]$.

While the failure mechanism in longitudinal compression for NCF reinforced composites is similar to that for UD composites, i.e. fibre kinking [10,11], the mechanisms are different for matrix related failure in the transverse plane. Olsson [9] observed that a NCF reinforced composite laminate loaded in transverse tension either failed at the fibre bundle matrix interface (i.e. interbundle fracture) with a fracture plane close to the 1-2 plane, see Figure 2 bundle I, or inside the fibre bundles (i.e. intrabundle fracture), see Figure 2 bundle II. Longitudinal cracks in the 1-2 plane have been found in [12], where they are 
observed at the interface between the fibre bundle and matrix in NCF cross-ply materials under in-plane tensile loading.

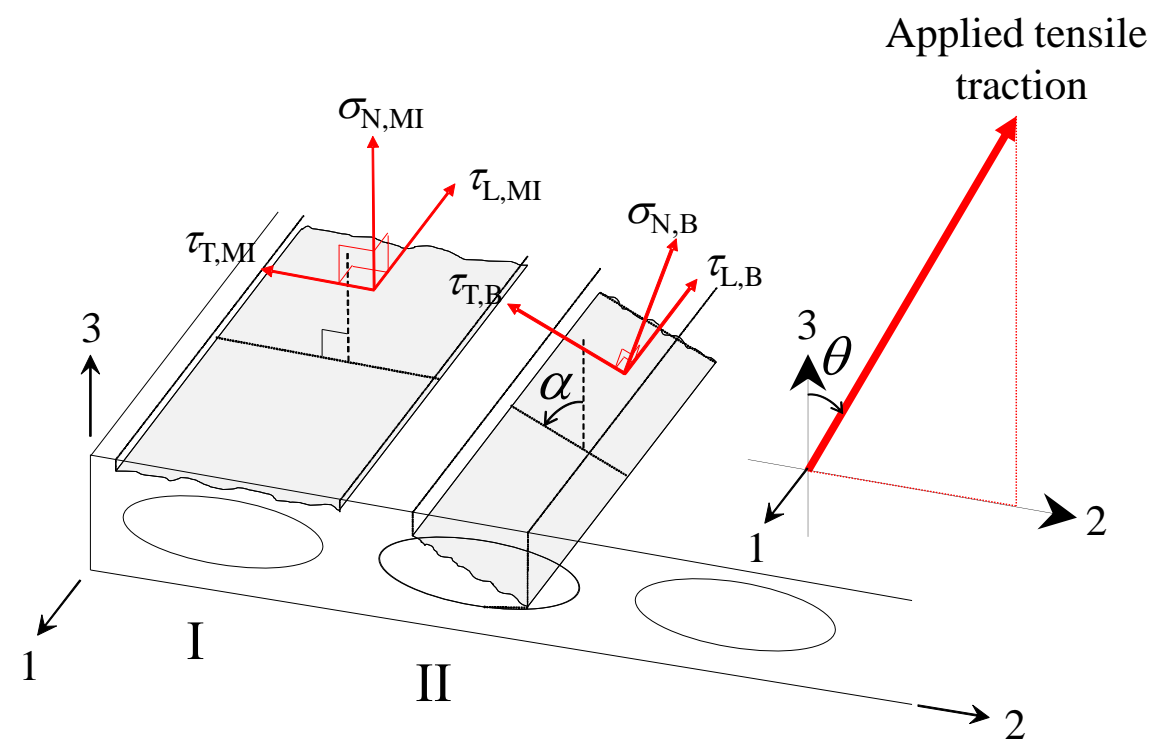

Figure 2. Fracture planes within a NCF lamina. Interbundle failure at Bundle I, with the fracture plane parallel to the NCF layer in the matrix. Intrabundle failure at Bundle II, with fracture plane inside the fibre bundle.

\section{Failure prediction of NCF reinforced composites}

The proposed analytical NCF model is developed in the standard framework for strength predictions of UD and NCF reinforced composites where stresses at the homogenised ply 
level are obtained by classical laminate theory. In particular, the model is designed to consider 3D stress states operating on the individual ply stresses.

In this section the development of a two modes failure model for NCF composites will be presented. However, existing relevant failure criteria for UD and orthotropic composites are first reviewed.

\section{State of the art failure criteria}

State of the art physically based failure criteria are available for UD reinforced composites, e.g. LaRC05 [5]. With such failure criteria it is possible to capture the failure behaviour, onset of failure, mode of failure and angle of the fracture plane within a given ply. This is of great importance if subsequent damage growth analyses are to be performed. The LaRC05 criteria performed well in the second world wide failure exercise [13] for UD laminates, and the material parameters it uses are measurable from standard tests. Since many of the failure mechanisms in NCF reinforced composites are similar to those in UD composites, the LaRC05 criteria provide good foundation for the development for NCF criteria. 
For fibre reinforcements in all directions, e.g. 3D reinforced textile composites, a set of physically based criteria developed by Juhasz et al. [14] exists that can predict either fibre failure or matrix failure in the composite. This set of criteria was not originally developed for NCF reinforced composites and cannot distinguish between different matrix dominated failure modes observed in the transverse plane. The Juhasz' criteria were later proposed for NCF reinforced composites in the FALCOM project [15]. However, no general acceptance for their use has yet been established. This is mainly due to the large number of strength parameters required and the difficulty to measure them.

To address the lack of a set of physically based failure criteria for NCF reinforced composites a new set of criteria based on the LaRC05 criteria is suggested. The set of criteria is developed to capture the non transversely isotropic nature of NCF composites as discussed above.

\section{LaRC05}

The LaRC05 failure criteria are based on three different failure modes; intralaminar matrix failure, fibre kinking, and fibre tensile failure.

Intralaminar matrix failure prediction in LaRC05 [5] considers the transverse shear, $\tau_{\mathrm{T}}$, longitudinal shear, $\tau_{\mathrm{L}}$, and the normal stress, $\sigma_{\mathrm{N}}$, on the fracture plane in Figure 3 . The 
strength values consider the in-situ effect, denoted with superscript 'is' in Eq. (1), and are based on the transverse tensile strength, $Y_{\mathrm{T}}$, transverse shear strengths, $S_{\mathrm{T}}$, and longitudinal shear strength, $S_{\mathrm{L}}$, as well as the fracture angle for pure transverse compression, $\alpha_{0}$.

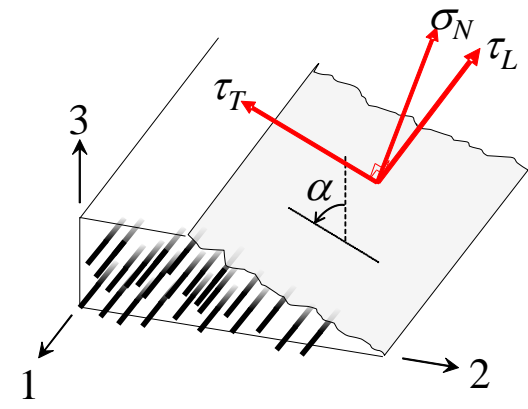

Figure 3. Tractions acting on the fracture plane for intralaminar matrix failure in unidirectional (UD) composites.

Matrix failure, $F I_{\mathrm{M}}$, is predicted when,

$$
F I_{\mathrm{M}}=\left(\frac{\tau_{\mathrm{T}}}{S_{\mathrm{T}}^{\text {is }}-\eta_{\mathrm{T}} \sigma_{\mathrm{N}}}\right)^{2}+\left(\frac{\tau_{\mathrm{L}}}{S_{\mathrm{L}}^{\text {is }}-\eta_{\mathrm{L}} \sigma_{\mathrm{N}}}\right)^{2}+\left(\frac{\left\langle\sigma_{\mathrm{N}}\right\rangle_{+}}{Y_{\mathrm{T}}^{\text {is }}}\right)^{2}=1
$$

where $\langle x\rangle$ denotes the Macauley bracket. 


$$
\langle x\rangle_{+}=\left\{\begin{array}{ll}
x, & x>0 \\
0, & x \leq 0
\end{array}, \text { or } \quad\langle x\rangle_{-}=\left\{\begin{array}{ll}
0, & x \geq 0 \\
x, & x<0
\end{array} .\right.\right.
$$

For intralaminar matrix failure, the transverse shear strength, $S_{\mathrm{T}}$, is related to the transverse compressive strength, $Y_{\mathrm{C}}$, as

$$
S_{\mathrm{T}}=Y_{\mathrm{C}} \cos \left(\alpha_{0}\right)\left(\sin \left(\alpha_{0}\right)+\frac{\cos \left(\alpha_{0}\right)}{\tan \left(2 \alpha_{0}\right)}\right) .
$$

The LaRC05 criteria capture fibre kinking or splitting using failure criteria applied to the stresses transformed into the kinking plane. Kinking, or splitting, occurs in a coordinate frame with the 1-direction parallel to the misaligned fibres and the tractions on this plane are denoted with the superscript $\mathrm{m}$. The stresses are calculated in this reference system through transformation. The failure mode, kinking or splitting, is distinguished by the magnitude of the compression stress along the fibres; if $\sigma_{11}<-X_{\mathrm{C}} / 2$ then kinking occur and if $\sigma_{11} \geq-X_{\mathrm{C}} / 2$ then splitting occur,

$$
F I_{\text {KINK }}=F I_{\text {SPLIT }}=\left(\frac{\tau_{23}^{\mathrm{m}}}{S_{\mathrm{T}}^{\text {is }}-\eta_{\mathrm{T}} \sigma_{22}^{\mathrm{m}}}\right)^{2}+\left(\frac{\tau_{12}^{\mathrm{m}}}{S_{\mathrm{L}}^{\text {is }}-\eta_{\mathrm{L}} \sigma_{22}^{\mathrm{m}}}\right)^{2}+\left(\frac{\left\langle\sigma_{22}^{\mathrm{m}}\right\rangle_{+}}{Y_{\mathrm{T}}^{\text {is }}}\right)^{2}=1
$$

where $X_{\mathrm{C}}$ is the compressive strength in the fibre direction. 
Fibre failure in tension occurs when the stress in the fibres exceeds the tensile strength,

$$
F I_{\mathrm{FT}}=\frac{\left\langle\sigma_{11}\right\rangle_{+}}{X_{\mathrm{T}}}=1 .
$$

Juhasz' criteria for 3D fibre reinforced polymer composites.

A set of failure criteria for 3D reinforced composites, with orthotropic properties, has been proposed by Juhasz et al. [14]. The criteria were developed for composites with high in-plane fibre density and additional out of plane fibre reinforcements. The strength model distinguishes between Fibre Failure (FF) and Inter Fibre Failure (IFF) (cf. intralaminar matrix fracture for LaRC05). For loads applied close to one of the axes with parallel fibres FF is evaluated, and when loaded at an off-axis fibre angle, $\theta>2^{\circ}$, IFF is evaluated. The IFF criterion is based on strength parameters that are interpolated from the strengths, $Y_{\mathrm{T} \mid \mathrm{C}}$ and $Z_{\mathrm{T} \mid \mathrm{C}}$ for the composite. The model is based on a simple parabolic criterion proposed by Cuntze et al. [16]. However, the basic strengths need to be adjusted and are obtained by fitting data to experimental results.

The parabolic criterion must be evaluated for a number of angles in the 2-3-plane in order to find the critical plane. This is done by looping over a number of angles from 0 to 180 degrees. The angle that maximises the failure index, $F I$, is the fracture plane angle. 


$$
\begin{gathered}
F I_{\text {IFF, Juhasz }}=\frac{\left\langle\sigma_{\mathrm{N}}\right\rangle_{+}}{\left|\sigma_{\mathrm{N}}\right|}\left[\left[\left(1-p^{(+)}\right)^{2}\left(\frac{\sigma_{\mathrm{N}}}{Y_{\mathrm{N} \mid \mathrm{T}}}\right)^{2}+\left(\frac{\tau_{\mathrm{T}}}{S_{\mathrm{T} \mid \mathrm{T}}}\right)^{2}+\left(\frac{\tau_{\mathrm{L}}}{S_{\mathrm{L}}}\right)^{2}\right]^{1 / 2}+p^{(+)} \frac{\sigma_{\mathrm{N}}}{Y_{\mathrm{N} \mid \mathrm{T}}}\right] \\
+\frac{\left\langle\sigma_{\mathrm{N}}\right\rangle_{-}}{\left|\sigma_{\mathrm{N}}\right|}\left[\left[\left(p^{(-)}\right)^{2}\left(\frac{\sigma_{\mathrm{N}}}{Y_{\mathrm{N} \mid \mathrm{C}}}\right)^{2}+\left(\frac{\tau_{\mathrm{T}}}{S_{\mathrm{T} \mid \mathrm{C}}}\right)^{2}+\left(\frac{\tau_{\mathrm{L}}}{S_{\mathrm{L}}}\right)^{2}\right]^{1 / 2}+p^{(-)} \frac{\sigma_{\mathrm{N}}}{Y_{\mathrm{N} \mid \mathrm{C}}}\right]=1
\end{gathered}
$$

The strengths for the failure plane used in the failure index calculations are calculated according to:

$$
\begin{aligned}
& Y_{\mathrm{N} \mid \mathrm{C}, \mathrm{T}}=\tilde{Y}_{\mathrm{C}, \mathrm{T}} \cos ^{2} \alpha+\tilde{Z}_{\mathrm{C}, \mathrm{T}} \sin ^{2} \alpha+\tilde{S}_{\mathrm{yz}}|\sin 2 \alpha| \\
& S_{\mathrm{L}}=\tilde{S}_{\mathrm{xy}}|\cos \alpha|+\tilde{S}_{\mathrm{xz}}|\sin \alpha| \\
& S_{\mathrm{T} \mid \mathrm{C}, \mathrm{T}}=\frac{1}{2}\left(\tilde{Y}_{\mathrm{C}, \mathrm{T}}+\tilde{Z}_{\mathrm{C}, \mathrm{T}}\right)|\sin 2 \alpha|+\tilde{S}_{\mathrm{yz}}
\end{aligned}
$$

where $\tilde{X}_{\mathrm{C}, \mathrm{T}}, \tilde{Y}_{\mathrm{C}, \mathrm{T}}, \tilde{Z}_{\mathrm{C}, \mathrm{T}}, \tilde{S}_{\mathrm{xy}}, \tilde{S}_{\mathrm{yz}}$ and $\tilde{S}_{\mathrm{xz}}$ are the fitted basic strengths.

For determination of all the 11 (9 strengths and $\left.p^{(+,-)}\right)$parameters needed in the set of criteria extensive material testing on coupon level is required together with curve fitting for finding the adjusted strength values used in the criteria. 


\section{Development of the failure criteria for NCF based on a two modes approach}

In the LaRC05 criteria [5] for intralaminar transverse failure in UD composites, Eq. (1), it is assumed that the transverse shear strength $S_{\mathrm{T}}$ is independent of the fracture plane angle. Hence, the compressive strength $Y_{\mathrm{C}}$ is independent on the fracture plane. Also, the normal stress $\sigma_{\mathrm{N}}$ acting on the fracture plane is evaluated against the tensile strength $Y_{\mathrm{T}}$ without any dependence on the fracture plane angle because of the transverse isotropy assumed for UD composites. Here a model for NCF reinforced composites based on the LaRC05 criteria [5] is proposed. The proposed model considers the transverse strength dependence on the direction of the load.

Two modes model for transverse NCF reinforced composite failure.

To address the two different failure modes in transverse tension as illustrated in Figure 2, two failure criteria are proposed. The first criterion evaluates the stress components against failure on a plane normal to the layer thickness, see Figure 2 bundle I, independently of how the stress is applied. On this fracture surface, called Matrix Interface (MI) between fibre bundle and matrix, the out of plane strength, $Z_{\mathrm{T}}$, is considered,

$$
F I_{\mathrm{M}, \mathrm{MI}}=\left(\frac{\tau_{\mathrm{T}, \mathrm{MI}}}{S_{\mathrm{T}, \mathrm{MI}}}\right)^{2}+\left(\frac{\tau_{\mathrm{L}, \mathrm{MI}}}{S_{\mathrm{L}, \mathrm{MI}}}\right)^{2}+\left(\frac{\sigma_{\mathrm{N}, \mathrm{MI}}}{Z_{\mathrm{T}}}\right)^{2}=1 \text {, if } \sigma_{\mathrm{N}}>0 .
$$


Here, the shear strength $S_{\mathrm{T}, \mathrm{MI}}$ and $S_{\mathrm{L}, \mathrm{MI}}$ are equal to the interlaminar shear strength (ILSS),

$$
S_{\mathrm{T}, \mathrm{MI}}=S_{\mathrm{L}, \mathrm{MI}}=I L S S \text {. }
$$

The second criterion for failure within the fibre bundle, corresponding to the original LaRC05 equation, Eq. (1), is denoted with the subscript B, see Figure 2 II,

$$
F I_{\mathrm{M}, \mathrm{B}}=\left(\frac{\tau_{\mathrm{T}, \mathrm{B}}}{S_{\mathrm{T}}-\eta_{\mathrm{T}} \sigma_{\mathrm{N}}}\right)^{2}+\left(\frac{\tau_{\mathrm{L}, \mathrm{B}}}{S_{\mathrm{L}}-\eta_{\mathrm{L}} \sigma_{\mathrm{N}}}\right)^{2}+\left(\frac{\left\langle\sigma_{\mathrm{N}, \mathrm{B}}\right\rangle_{+}}{Y_{\mathrm{T}}}\right)^{2}=1 .
$$

Where the shear strength $S_{\mathrm{T}}$ is based on $Y_{\mathrm{C}}$ as:

$$
S_{\mathrm{T}}=Y_{\mathrm{C}} \cos \left(\alpha_{0}\right)\left(\sin \left(\alpha_{0}\right)+\frac{\cos \left(\alpha_{0}\right)}{\tan \left(2 \alpha_{0}\right)}\right) \text {, }
$$

and where the friction parameters $\eta_{\mathrm{T}}$ and $\eta_{\mathrm{L}}$ are related to the shear strengths $S_{\mathrm{L}}$ and $S_{\mathrm{T}}$ as:

$$
\frac{\eta_{\mathrm{T}}}{S_{\mathrm{T}}}=\frac{\eta_{\mathrm{L}}}{S_{\mathrm{L}}}
$$

Consequently, transverse failure is evaluated as the maximum of $F I_{\mathrm{M}, \mathrm{MI}}$ and $F I_{\mathrm{M}, \mathrm{B}}$ for all angles.

$$
F I_{\mathrm{M}}=\max \left(F I_{\mathrm{M}, \mathrm{MI}}, F I_{\mathrm{M}, \mathrm{B}}\right)
$$




\section{Meso-mechanical finite element model of an NCF reinforced composite}

This part presents the numerical model, developed and used for validation of the analytical model. The description of the numerical work is divided in two sections, where the first presents the Finite Element (FE) model concerning modelling assumptions, material data and failure criteria. The subsequent section describes the numerical approach with all essential steps from preprocessing to the plotting of failure envelopes.

\section{Representative volume element}

The meso-architecture of fibre bundles for an NCF in the transverse plane is irregular due to preforming, nesting of plies, etc. as can be seen in Figure 1(b). The most realistic RVE should therefore contain several fibre bundles with irregular shape. The present work studies the interaction between the bundle and surrounding matrix on a fundamental level. It is of interest to study the effect of varied bundle parameters. To achieve this in a simplified way, it is therefore convenient to assume a repetitive meso-architecture in the sense that all bundles contains the same amount of fibres and have the same shape. This approach leads to a rectangular RVE containing one impregnated fibre bundle with surrounding matrix as can be seen in Figure 4. Periodic boundary conditions are applied in the transverse directions, in the 2-3 plane, further discussed in the Numerical procedure section. 
The shape of the bundle is governed mainly by the volume it accommodates, i.e. almost rectangular since the bundle volume fraction is $87 \%$. This volume fraction is based on an NCF composite fibre volume fraction of $56 \%$ and a fibre volume fraction within the bundle of $65 \%$. Fibre bundles in NCF reinforced composites are typically closer to each other in the out of plane direction than in plane. Positioning of the super ellipse within the RVE is based on this observation, giving the nominal geometry shown in Figure 4(a). To account for imperfections in bundle geometry and imperfections introduced by the stitch yarn, a perturbed geometry is also investigated as shown in Figure 4(b).
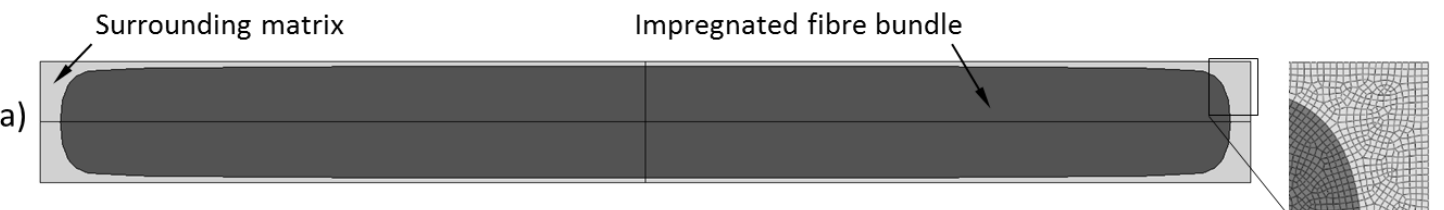

b)

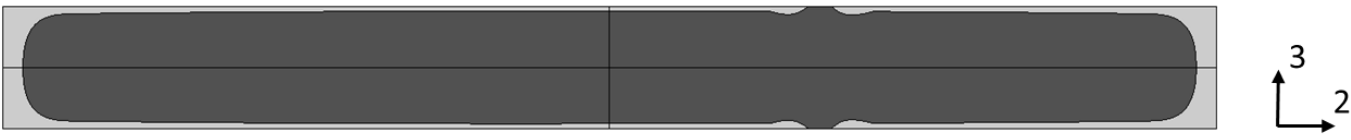

Figure 4. (a) RVE model of an NCF reinforced composite with "nominal" geometry $\left(V_{f}=0.56\right)$. The dark grey part represents a fibre bundle with 12,000 fibres (nominal fibre volume fraction in the bundle of $V_{f b}=65 \%$ ) and the light grey section represents the matrix material. (b) Similar RVE model as in (a) but with a "perturbed" geometry. 
Since no variations of the meso geometry in the 1-direction are considered in this work, generalized plane strain conditions are assumed which reduce the computational cost. The RVE is modelled with a shape factor of 10 between width and height, which is close to the observed aspect ratio of 13 for this type of NCF [6]. Two other shape factors, 6.7 and 13.3 have been verified with low impact on the results. Mesh convergence is achieved with a discretization of $600 \times 60$ elements, which is equivalent to $5 \mu \mathrm{m}$ elements for an RVE size of $3 \times 0.3 \mathrm{~mm}$. First order thermo-mechanical elements are used with 4 integration points per quadrilateral element and one integration point per triangular element.

Elastic and thermal properties for fibre and matrix are similar to those reported by Marklund et al. [6] and are presented in Table 2. Homogenized elastic and thermal properties for the fibre bundle are calculated using Halpin-Tsai and the rule of mixtures. The Halpin-Tsai model has been shown by Marklund et al. [17] to accurately predict the transverse stiffness for the high fibre volume fractions in the bundle. 
Residual stresses due to post-curing thermal loading is included by performing a load step with a temperature decrease $\Delta \mathrm{T}$ of $-160^{\circ} \mathrm{C}$. These residual stresses are added to the stresses caused by mechanical loading.

\section{Failure analysis}

Two different failure criteria are implemented in the meso-micromechanical FE model. Intrabundle matrix failure, is predicted using Eq. (1) in the impregnated fibre bundle and its implementation is similar to that of the analytical model. Failure initiation in the surrounding matrix material is predicted with the Raghava yield criterion [18]. The Raghava criterion is based on von Mises plasticity theory and adapted to polymers by including hydrostatic pressure dependence. The Raghava yield criterion is written as

$$
\left(\sigma_{1}-\sigma_{1}\right)^{2}+\left(\sigma_{2}-\sigma_{3}\right)^{2}+\left(\sigma_{3}-\sigma_{1}\right)^{2}+6(C-T) \sigma_{m}=2 C T
$$

where

$$
\sigma_{m}=\frac{\left(\sigma_{1}+\sigma_{2}+\sigma_{3}\right)}{3}
$$

$C$ and $T$ are the absolute values of the compressive and tensile yield stresses of the matrix material given as $Y_{\mathrm{C}}$ and $Y_{\mathrm{T}}$ in Table 1. $\sigma_{1}, \sigma_{2}$ and $\sigma_{3}$ are the principal stresses in the surrounding matrix material. 
Strength data for the fibre bundle and surrounding matrix are presented in Table 1. Strength data for the fibre bundle were obtained from tests performed on a UD composite equivalent to the fibre bundle in the considered NCF [6]. The reported compressive strength for this UD equivalence was lower than the compressive strength for the NCF reinforced composite, which is not feasible. Two different approaches have been used to mitigate this lack of data; i) a value of $200 \mathrm{MPa}$, which is equal to the value reported by Marklund et al. [6] for the bundle, ii) a value derived from the FE model to give a simulated NCF composite strength of $200 \mathrm{MPa}$ when bundle failure is critical. This is further discussed in the Compressive strength $Y_{\mathrm{c}}$ section below.

Failure initiation in the surrounding matrix due to tensile loading is assumed to correspond to the yield limit due to brittle fracture behaviour. Failure initiation in compression is also predicted by the yield limit. However, in this case the polymer may undergo large local plastic deformation before fracture occurs [19]. This approach is used for the main part of the work but a complementary analysis allowing for plastic deformation beyond the yield limit has also been performed and is described in the Effects of non-linear material response section. 


\section{Numerical procedure}

The numerical procedure is based on linear superposition of stresses for the generation of failure envelopes. Thus, it is assumed that the material response up to failure initiation is elastic. The main idea of this approach is computational efficiency such that variations can be studied in an efficient manner. A full description of the numerical procedure is given in [20]. Below a brief outline of the approach is presented for comprehension.

Pure strain loading for four fundamental load cases is applied to an RVE in the commercial FE program Abaqus. The stresses at each integration point of the RVE are read by a Fortran program. Homogenisation, superposition and failure evaluation are then performed. Failure envelopes can be plotted in Matlab or in the commercial FE postprocessing program Meta Post as contour damage plots.

Periodic boundary conditions are achieved in Abaqus by displacement constraints on nodal degrees of freedom in the form of equations, this is done using the keyword *EQUATION in the Abaqus input deck [21]. Opposite edges are coupled as shown in Figure 5(a) and applied strain on the RVE is coupled to displacement of the corner nodes 2-4 according to 


$$
\begin{aligned}
& \mathbf{u}_{T}=\mathbf{u}_{B}+\mathbf{u}_{3}-\mathbf{u}_{2}, \\
& \mathbf{u}_{R}=\mathbf{u}_{L}+\mathbf{u}_{3}-\mathbf{u}_{4},
\end{aligned}
$$

where index $T=$ Top, $B=$ Bottom, $L=$ Left and $R=$ Right, see Figure 5 . These coupling equations are defined with sets while assuring a consistent node numbering and positioning. Node 1 is the reference node and it has fixed boundary conditions. The coupling of periodic boundary conditions is done via formulation of sets, while ensuring a consistent numbering and positioning of opposite node pairs.
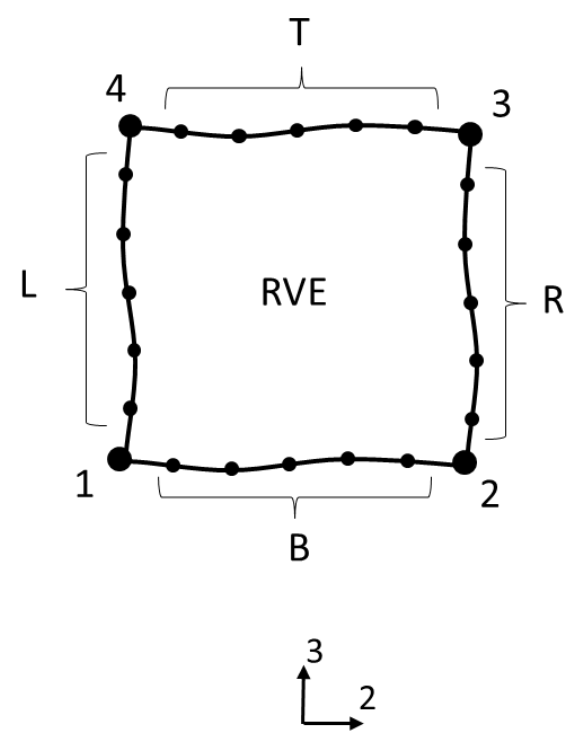
Figure 5. Schematic illustration of the RVE with periodic boundary conditions and node sets $L, T, R$ and $B$.

Thermal loading is applied as a coupled thermo-mechanical step. Boundary conditions of the corner nodes defined in the thermo-mechanical step, are such that the RVE is free to contract or expand. This stress state is added to the superposed stress state from mechanical loading.

Stress components for all integration points are linearly superposed to achieve all load combinations in the failure envelope which is presented in an xy-graph. This may be done for any combination of homogenised stress applied on the RVE, i.e. $\bar{\sigma}_{11}, \bar{\sigma}_{22}, \bar{\sigma}_{33}$ or $\bar{\tau}_{23}$

Failure envelopes for multi-axial loading may be produced by stepping along one axis in the xy-coordinate system until a failure index of 1 obtained. This is however not a robust method for arbitrary shaped failure curves that should be plotted in all four quadrants. The method used in this work is based on predefining a "failure map" domain and calculating the failure index for all load combinations within, see [20] for a full description. The failure curve is then obtained by interpolating these values in Matlab by 
the Contourc function and then plot this XY-data in normal fashion. The failure map is stored as a matrix with size

$$
m=\left(Y_{\max }-Y_{\min }\right) / Y_{\text {res }}+1 \quad ; \quad n=\left(X_{\text {max }}-X_{\text {min }}\right) / X_{r e s}+1,
$$

where $m$ is the number of rows and $n$ is the number of columns. $Y_{\text {res }}$ and $X_{\text {res }}$ refers to the resolution of each axis.

\section{Results and discussion}

In this section we first present strength predictions by the proposed NCF criteria in the analytical model and compare those with predictions made with the transversely isotropic LaRC05 criteria and the orthotropic Juhasz' criteria. Following this, we validate the proposed failure criteria for a HTS/RTM6 NCF composite by comparing its predicted failure envelope in the transverse plane with the failure envelope predicted by the numerical RVE model. Note that the numerical model considers strength data for the individual constituents, i.e. the fibre bundle and the matrix material, whereas the analytical model considers strength data for the UD NCF composite material.

\section{Strength predictions and failure envelopes}

Predictions with the proposed analytical NCF criteria and the LaRC05 criteria based on $Y_{\mathrm{T}}$ and $Z_{\mathrm{T}}$ properties are presented in Figure 6. The stress is applied at an off-axis angle $\theta$ 
from the 3-axis towards the 2-axis, as illustrated in Figure 2. Due to the assumption of transverse isotropy predictions made with the LaRC05 criteria are insensitive to the angle $\theta$ and predict a constant strength value, either $Y_{\mathrm{T}}$ or $Z_{\mathrm{T}}$ (plotted as two horizontal lines in the graph). These horizontal lines constitute the upper and lower bounds for the proposed NCF criteria.

The proposed analytical NCF criteria evaluate both the matrix interface criterion, $F I_{\mathrm{M}, \mathrm{MI}}$, Eq. (10), and the matrix bundle criterion, $F I_{\mathrm{M}, \mathrm{B}}$, Eq. (12), at all loading conditions. The lowest value of $F I_{\mathrm{M}, \mathrm{MI}}$ and $F I_{\mathrm{M}, \mathrm{B}}$, Eq. (15), determines the transverse matrix dominated failure mode for the current loading. The graph shows that the upper bound from the LaRC05 criteria, assigning transverse strength by $\mathrm{Y}_{\mathrm{T}}$, overestimates the strength in the out of plane direction for NCF reinforced composites at loading angles $<30^{\circ}$. However, for loading angles $\geq 35^{\circ}$ strength predicted by the LaRC05 and the NCF criteria both are identical and accurately predict intrabundle failure in the NCF composite. In contrast, assigning $\mathrm{Z}_{\mathrm{T}}$ as the transverse strength in the LaRC05 criteria strongly underestimate the strength in the plane, and only provide acceptable prediction of strength (i.e. interbundle strength) for very small off-angles to the out-of-plane direction. 


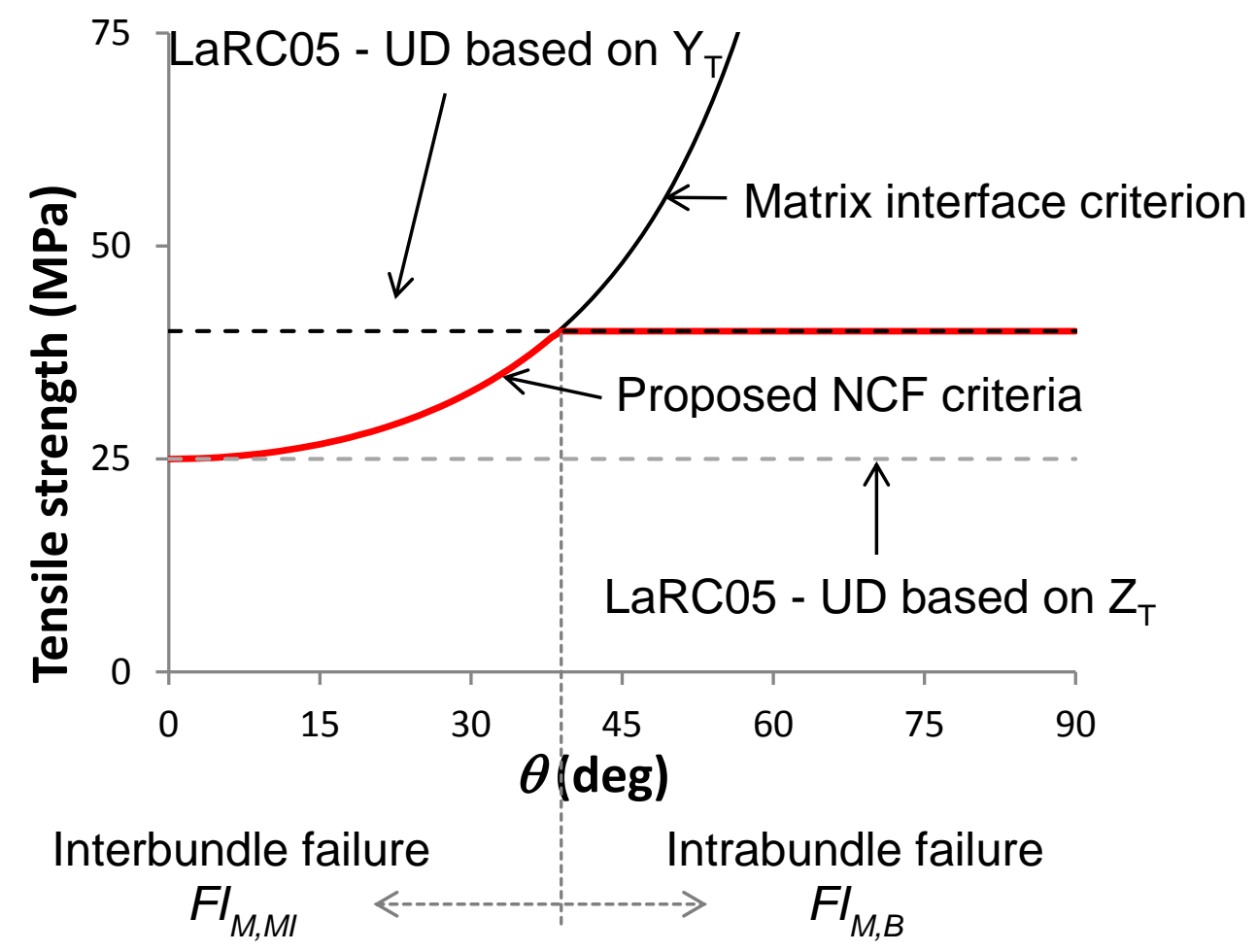

Figure 6. Predicted strength for a tensile stress at an angle $\theta$. Load applied as in Figure 2.

Predicted failure envelope in the $\sigma_{22}-\sigma_{33}$ plane by the proposed analytical NCF criteria are plotted in Figure 7 together with predictions made with the LaRC05 criteria and experimental data from Olsson [9]. At each corner of the envelope, the fracture plane angle $\alpha$ changes, see Figure 7. The LaRC05 criteria overestimate the strength in the 3- 
direction due to the assumption of transverse isotropy. Both criteria underestimate the compressive strength in the 3-direction. This is due to the fact both criteria use the compressive $Y$-strength for calculation of the transverse shear strength, see Eqs. (3) and (13).

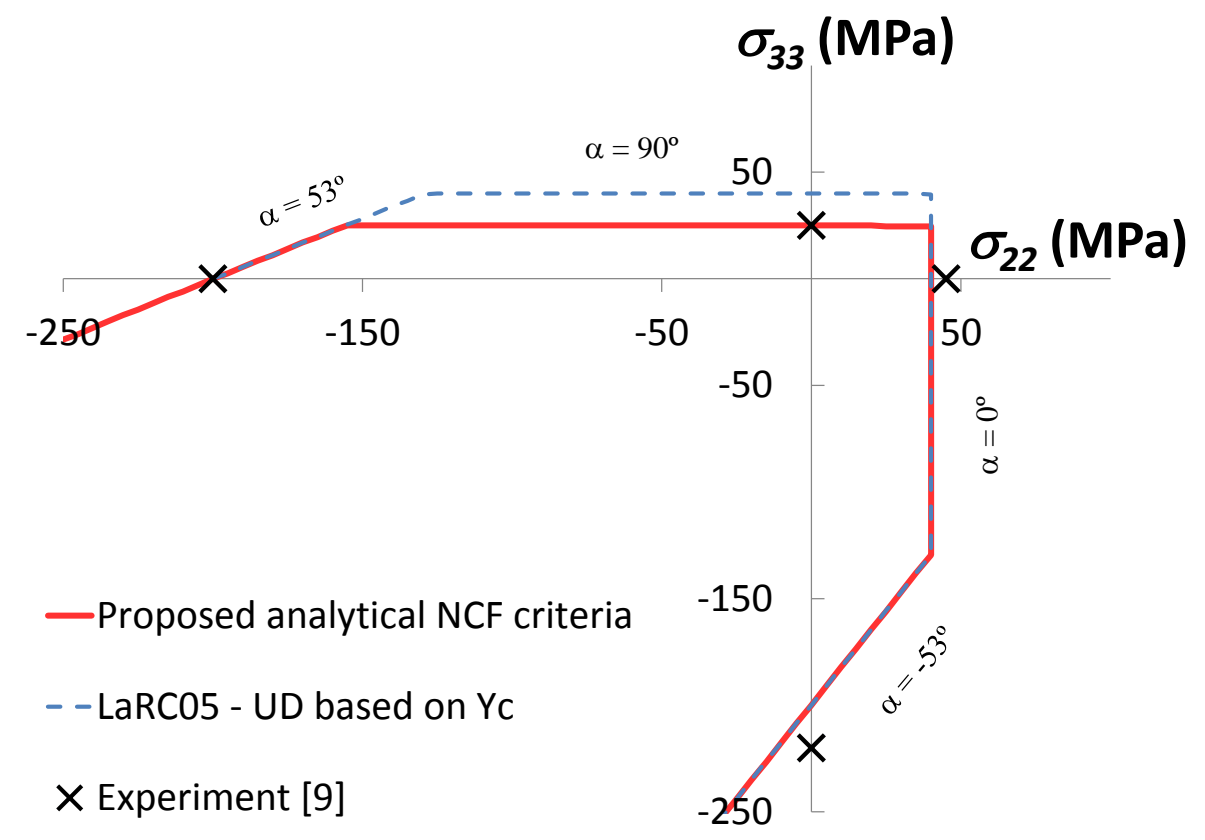

Figure 7. Predicted failure envelope in the $\sigma_{22}-\sigma_{33}$ plane for the proposed analytical NCF criteria and the LaRC05 criteria based on $Y_{\text {c }}$. 
Predictions with a fracture plane $\alpha=90^{\circ}$ corresponds to the interbundle failure mode, while failure at other fracture plane angles corresponds to the intrabundle failure mode, i.e. failure inside the fibre bundle.

The proposed analytical NCF criteria are compared with the orthotropic criteria proposed by Juhasz and co-workers for loadings according to Figure 2 . The proposed analytical NCF criteria are dependent on the tensile strengths $Y_{\mathrm{T}}$ and $Z_{\mathrm{T}}$, whereas to the Juhasz' criteria are dependent on all strengths; tensile, compressive and shear in all directions. In particular, the shear strength is shown to strongly influence strength predictions with the Juhasz' criteria for off axis angles in the 2-3 plane, as illustrated in Figure 8. The proposed analytical NCF criteria sets the transverse shear strength equal to the interlaminar shear strength of the composite, according to Eq. (11). Both criteria predict the same on-axis strengths (i.e. $\theta=0^{\circ}$ or $90^{\circ}$ ), see Figure 8. At off-axis angles predictions with Juhasz' criteria become very sensitive to the fitted shear strength value, whereas the proposed analytical NCF criteria provides a smooth transition independent of the shear strength as shown in Figure 8. 


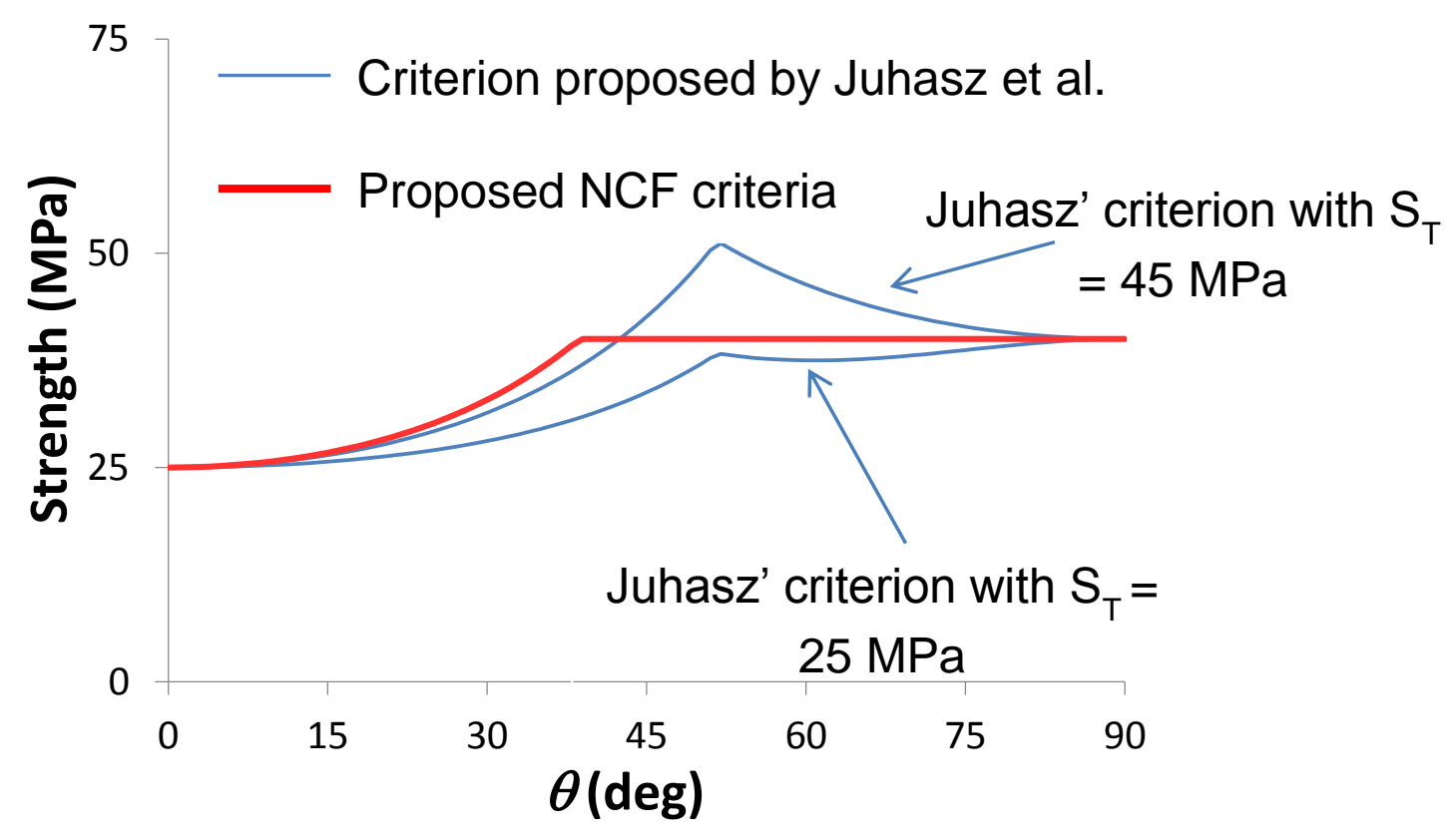

Figure 8. Application of the stress at an off axis angle $\theta$ from the $Z$-axis to the $Y$-axis.

Predicted strength for a tensile stress at an angle $\theta$. Proposed analytical NCF criteria and the criteria proposed by Juhasz et al. [14].

The proposed analytical NCF criteria are validated against predictions made with the numerical meso-micromechanical model. Predicted failure envelopes for the $\sigma_{22}-\sigma_{33}$ plane are depicted in Figure 9(a). In the figure, the failure envelope predicted by the proposed analytical NCF criteria considering material data for the NCF reinforced composite, is plotted with those predicted by the numerical meso-micromechanical RVE 
model, which considers only material data for the constituents (i.e. fibre bundle and matrix material). All material data are presented in Table 1. In Figure 9(a) the numerical RVE model provides two failure envelopes, one for intrabundle driven failure and one for matrix yielding initiated failure. Consequently, transverse failure of the NCF composite is predicted by the numerical model to be initiated by the failure mode triggered at the lowest stress state for any given load. That is, the failure envelope predicted by the numerical RVE model that is closest to the origin in Figure 9(a) will initiate transverse failure of the composite. Examining the failure envelopes labelled "RVE model - Intrabundle failure" and "RVE model - Matrix yielding" in Figure 9(a) intrabundle failure is predicted to initiate failure for biaxial tensile stress states, whereas for compression dominated stress states failure is predicted to be driven by matrix yielding in the matrix material surrounding the fibre bundle. This confirms the mode switch predicted by the analytical NCF model.

The agreement for pure tensile loads is good between the proposed analytical NCF criteria and the numerical RVE model, including matrix yielding, thermal effects and geometric perturbations. A sensitivity analysis of the influence of these parameters on the numerical predictions is presented in the following section. As mentioned above, for moderate to 
high compressive loads the numerical RVE model predicts yielding of the surrounding matrix as the failure mode. However, yielding is predicted to be very localised in the matrix material. Following this local matrix yielding, intrabundle failure is predicted to occur.

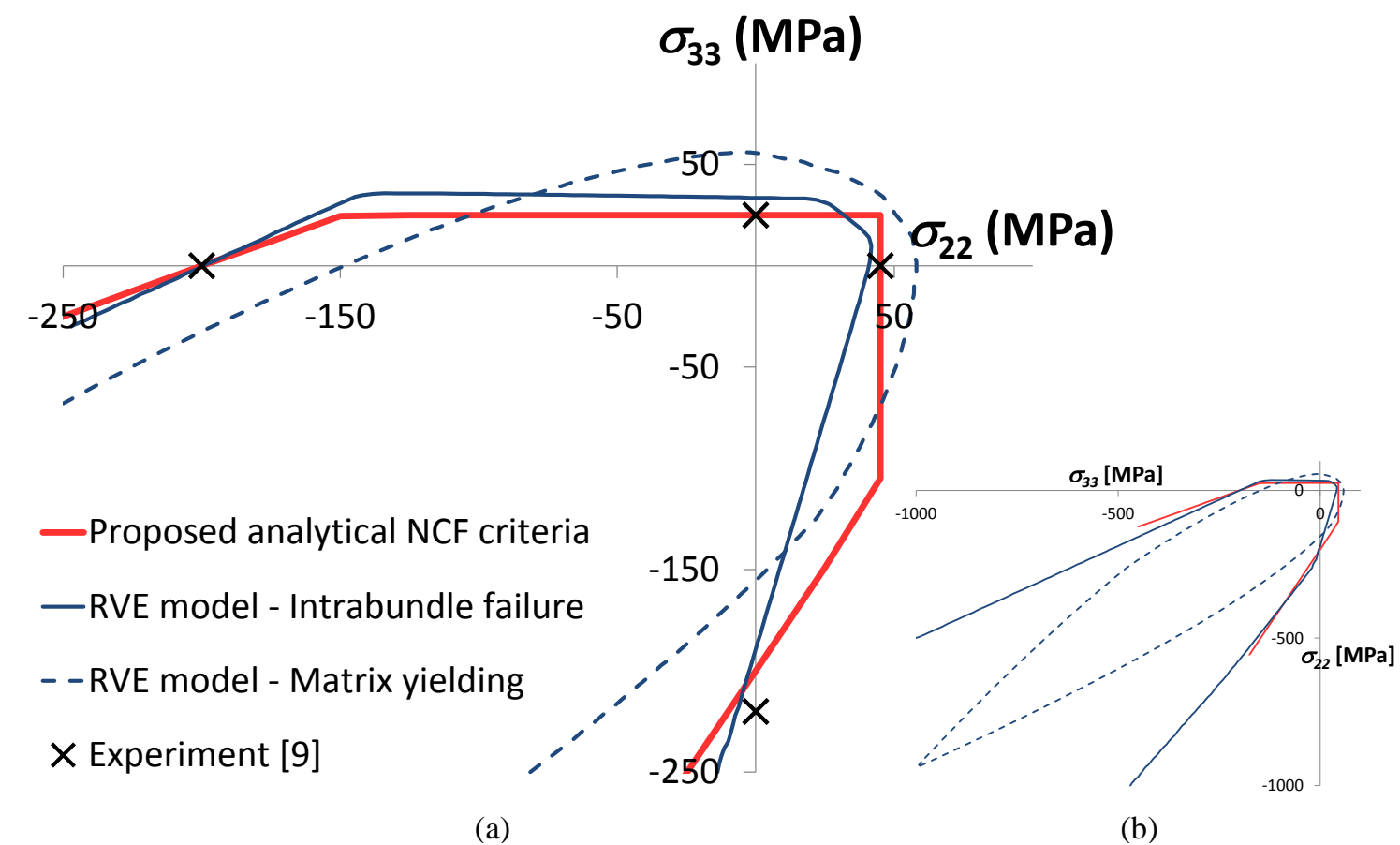

(a)

(b)

Figure 9. (a) Predicted failure envelope in the $\sigma_{22}-\sigma_{33}$ plane for the proposed analytical NCF criteria and the RVE model with its two constituents. (b) The RVE model predicts a closed failure envelope for the matrix yielding criteria under high compressive loads. 
The Raghava yield criterion used for matrix failure within the RVE model for the interbundle failure predicts a closed failure envelope, as illustrated in Figure 9(b), in contrast to both the proposed analytical NCF and the intrabundle failure criteria in the RVE model, which both predicts open failure envelopes. Experimental data for NCF reinforced composites under biaxial compressive stresses are not available. However, Kaddour and Hinton [13] reported that under pure hydrostatic pressure on UD Eglass/epoxy laminate the trend for the failure envelope is open. This finding is similar to the prediction shown in Figure 9(b) for the analytical NCF criteria and intrabundle failure in the RVE model.

\section{Sensitivity analysis of the meso-micromechanical RVE model}

Effects of selected parameters in the numerical model on failure prediction are assessed. The studied parameters are material data, including a non-linear response of the matrix material, thermal effects from cooling from manufacturing temperature to ambient temperature and geometric perturbation of the fibre bundle.

Effects of non-linear material response

The criterion used for the matrix in the meso-micromechanical RVE model is in fact a yield criterion and not a failure criterion. When applied in the model it will predict onset of plastic deformation within the matrix. For some stress combinations localised yielding 
of the matrix will occur before failure initiates in the fibre bundle. In a study by Marklund et al. [6] strain to failure between $30-50 \%$ was reported for the matrix material when loaded in uniaxial compression. The RTM6 matrix was reported to yield at approximately 3\% strain under this loading condition [6]. Consequently, the RTM6 matrix material can sustain substantial plastic deformation prior to failure. In order to analyse the influence of matrix yielding on failure initiation in the numerical model the non-linear material response for the RTM6 material reported by Marklund et al. [6] was introduced for the matrix material in the numerical RVE model. Non-linear analysis for an inplane compressive load case acting on the NCF RVE model was then performed, both for the nominal and perturbed geometry. Results show that intrabundle failure becomes critical when the total strain in the matrix is about $9.5 \%$, see Figure 10(a). At this point the equivalent plastic strain is about $4.5 \%$ and very localised as illustrated in Figure 10(b). Consequently, this localised yielding will not result in failure. Instead failure of the NCF composite will be governed by brittle intrabundle failure. Under the condition of elasticideally plastic behaviour of the matrix analyses performed imply that failure of the RVE will be governed by intrabundle failure preceded by highly localised plastic deformation in the surrounding matrix for most stress combinations in the second and fourth quadrants of the stress-plane in Figure 9(a). 
The failure index for the surrounding matrix in the non-linear simulations converges to a value slightly above unity, this is due the fact that different yield models are used in the meso-micromechanical model, that uses the Ragahva criterion, and the one implemented in Abaqus, that uses a Drücker-Prager model [21].

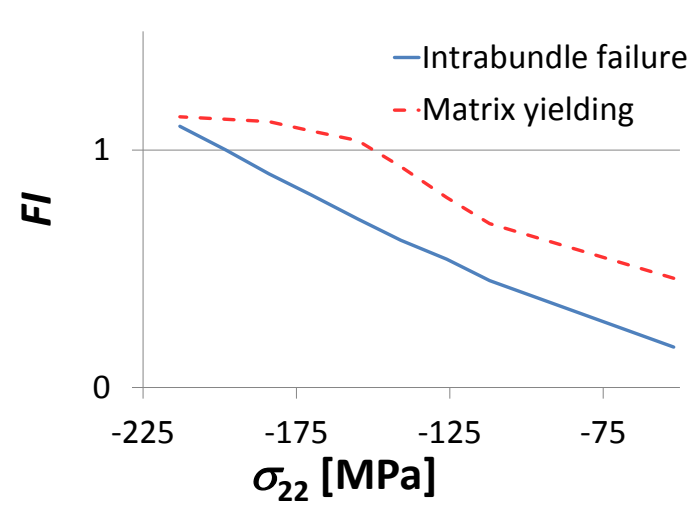

(a)

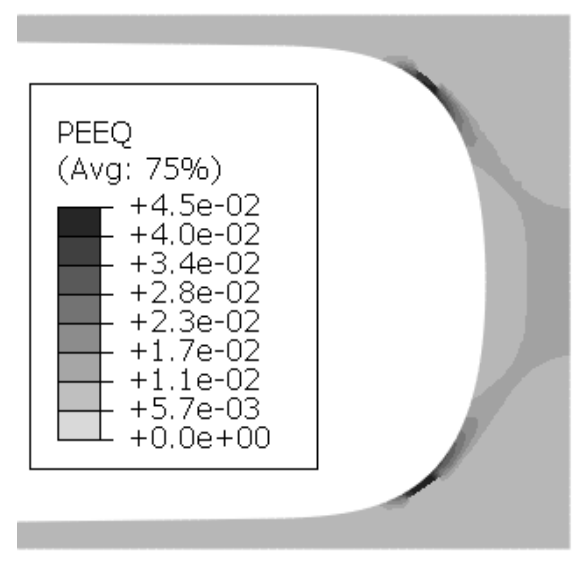

(b)

Figure 10. (a) Failure envelopes predict with the RVE with thermal effects and adjusted $Y_{c}$ for the perturbed geometry. (b) Equivalent plastic strain when both $F I_{M}$ and $F I_{B}$ equals I for the perturbed geometry. 


\section{Effects of thermal stresses on the RVE model}

Residual stresses from the temperature change during manufacturing are included in the RVE model as an additional thermal load case. This makes it possible to analyse how these stresses effects the failure initation in both fibre bundle and surrounding matrix. The failure envelopes for the perturbed geometry both with and without thermal effects are shown in Figure 11. The graph shows that thermal stresses strongly affect the transverse strength of the NCF reinforced composite. The strongest influence of the thermal residual stresses is found on the matrix yielding driven strength. For the fibre bundle the situation is different.

To understand the thermal effect on matrix yielding and intrabundle failure the stress states from the RVE model with and without thermal effects are compared. As a result of the thermal stresses arising from manufacture at elevated temperature the surrounding matrix material imposes compressive stresses on the fibre bundle. This explains the observed decrease at compressive loadings and increase at tensile loadings for the predicted intrabundle failure in Figure 11. The failure location for intrabundle failure is not affected by the thermal stresses and remains at the location of the geometric perturbation as shown in Figure 4. In contrast, the location of matrix yielding in the surrounding matrix changes due to thermal stresses. 


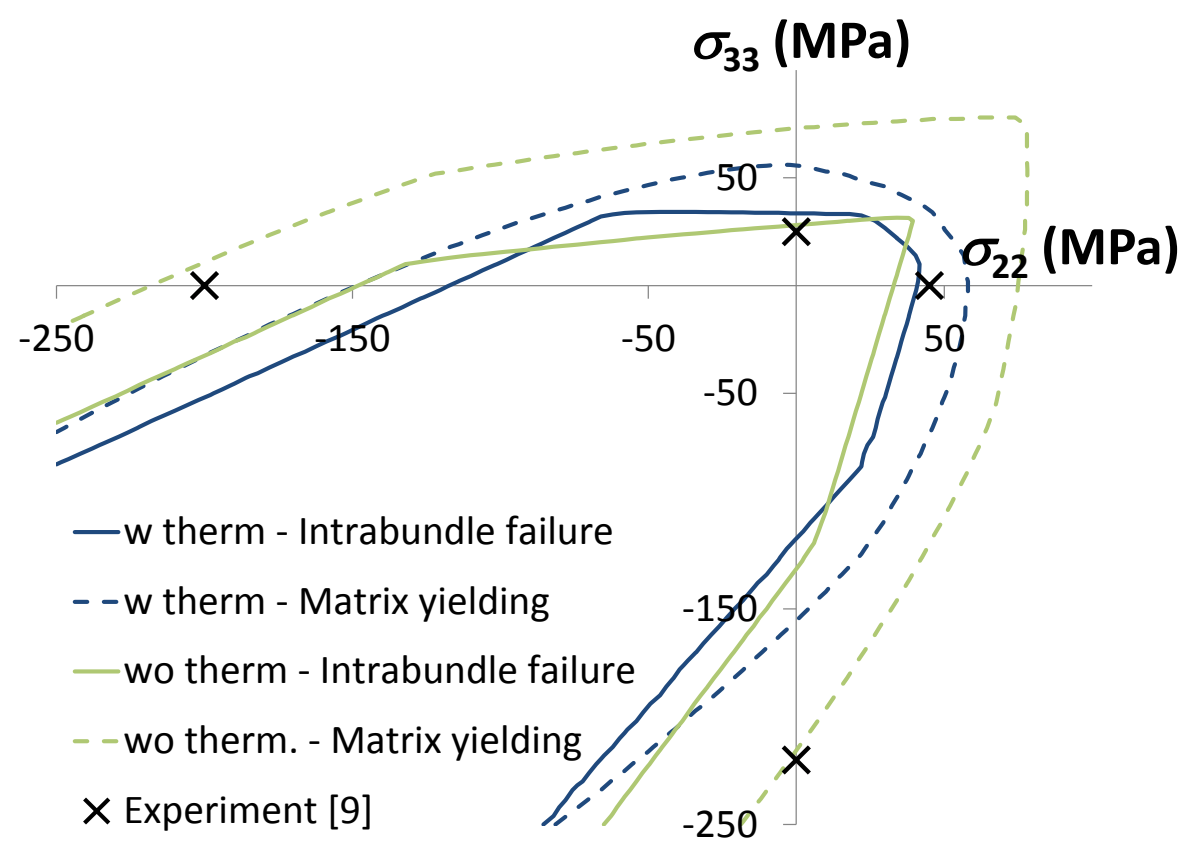

Figure I I. Failure envelopes predict with the Representative Volume Element (RVE) with and without thermal effects on the perturbed geometry.

Compressive strength $Y_{\mathrm{c}}$

A compressive strength $Y_{\mathrm{c}}$ is needed as a parameter both in the proposed analytical NCF criteria and the intrabundle failure in the RVE model. However, different compressive strengths are considered in the two models. In the analytical NCF criteria the compressive strength of the UD NCF composite is used, whereas the numerical RVE model considers the compressive strength of the bundle. Consequently, the compressive strength of the 
bundle must be higher than the strength for the UD NCF reinforced composite. This is not the case for the data reported by Marklund et al. [6]. In their paper they report on inaccurate test results for the bundle material, which explains this anomaly. Furthermore, corrections to the compressive strength value $Y_{\mathrm{c}}$ must consider influence of thermal residual stresses in the laminate, see Figure 11. In Figure 12, the predicted failure envelope for a compressive fibre bundle strength, $Y_{\mathrm{c}}$, of $200 \mathrm{MPa}$ (as reported in Table 1) is compared to the failure envelope adopting an adjusted compressive fibre bundle strength $Y_{\mathrm{c}}=310 \mathrm{MPa}$. The adjusted compressive fibre bundle strength value was attained from iterations to fit the in-plane compressive strength of $200 \mathrm{MPa}$ for the NCF reinforced composite. 


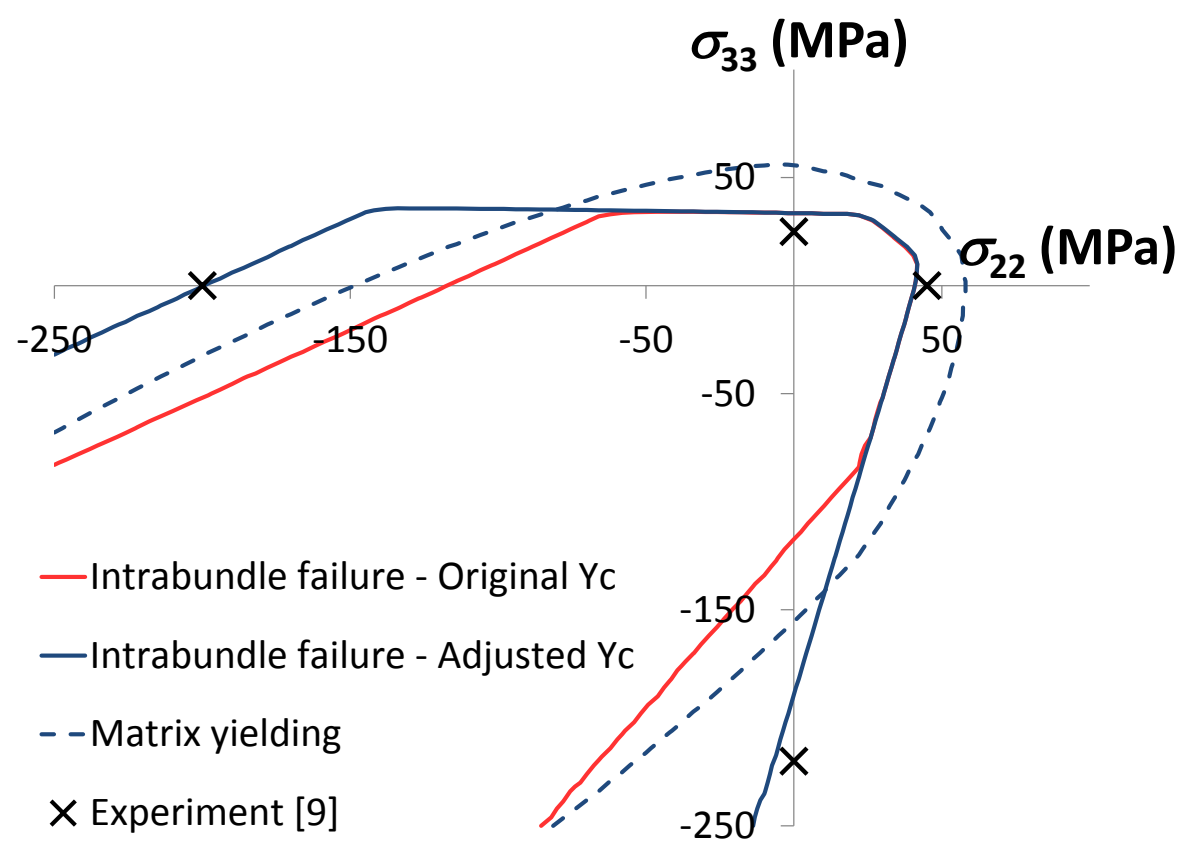

Figure I2. Failure envelopes predicted with the RVE model with and without an adjusted compressive strength, $Y_{c}$, of the fibre bundle to predict the correct failure when loaded with compressive stress in the 2-direction including thermal stresses and geometric perturbation.

Effect of geometric perturbation of the fibre bundle on orthotropy

With the RVE model of the fibre bundle and matrix it is possible to evaluate how the architecture of the NCF reinforcement affects the strength properties. Results of two different fibre bundle geometries are shown in Figure 13, one with a nominal fibre bundle geometry and one with a perturbed fibre bundle geometry, as depicted in Figure 4. Here it can be seen that the geometrical perturbation causes a decrease of the strength of the 
composite for the out of plane stresses where failure is governed by intrabundle failure. In contrast, the effect of fibre bundle geometry does not significantly affect the onset of matrix yielding in the composite.

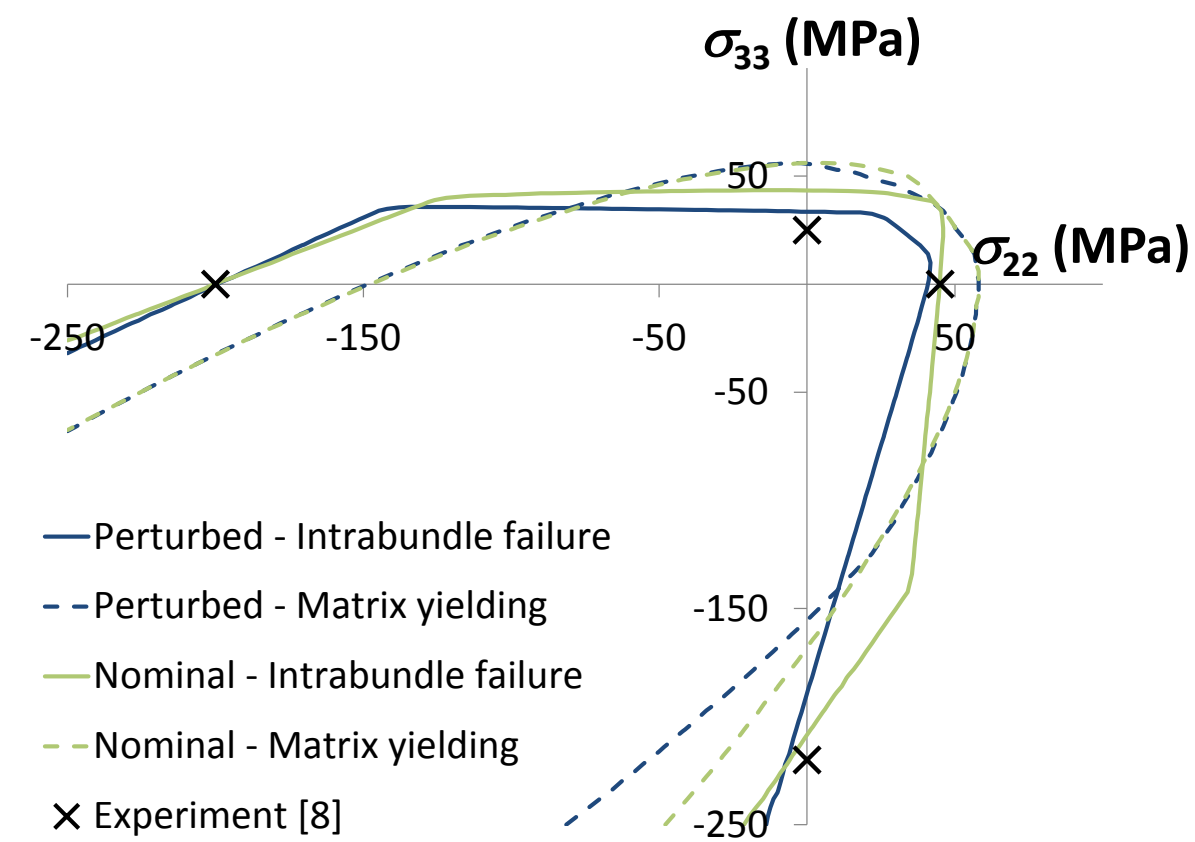

Figure 13. Failure envelopes predict with the Representative Volume Element (RVE) with thermal effects on the perturbed geometry and the nominal geometry using the corrected Yc value.

For the nominal geometry the failure locations are diffuse with large areas with approximately the same failure index for both the fibre bundle and the surrounding matrix 
for the $\sigma_{22}-\sigma_{33}$ envelope. For the perturbed geometry the locations undergoing plastic deformation in the surrounding matrix are still diffuse, but the intrabundle failure is localised to the perturbation.

Variations of the shape and position of fibre bundles within the NCF reinforced composite is similar to the modelled fibre bundle found in [6]. The shape variation and position close to another bundle creates local stress concentrations. The weft stitching yarn, crossing in between the tows, introduces similar stress concentrations and load introduction and can also affect the strength in the same way. The effect of stitching yarns is however not explicitly addressed in the current study.

\section{Conclusions}

Unlike unidirectional tape-based composites Non-Crimp Fabric reinforced composites are not transversely isotropic. To allow robust and efficient design with NCF reinforced composites accurate failure criteria must be developed. Here, we propose the first set of failure criteria designed to predict initiation of transverse failure in NCF reinforced composites. The failure criteria operate on the ply level considering its homogenised ply properties. 
The developed NCF composite failure criteria are founded on state-of-the-art failure models for intralaminar transverse failure of UD-composites. Analyses show that the proposed NCF criteria capture the orthotropic strength behaviour of the material and distinguish between intrabundle and interbundle failure modes.

Numerical experiments were performed to provide additional data for validation of the proposed NCF criteria. The numerical experiments addressed combined inplane and out-of-plane loading. The numerical RVE model considered two material phases; a homogenised representation of the NCF fibre bundle and the surrounding matrix material. Analytical predictions with the proposed NCF failure criteria show a general good agreement with the numerical predictions. In particular, the switch in failure mode from intrabundle to interbundle predicted by the analytical model, when loading changes from inplane to out-of-plane, is confirmed in the numerical experiments. Thus, the numerical model does not only provide means to validate the proposed NCF failure model but also provide deeper insight in the prevalent mechanisms governing failure of NCF composites exposed to transverse mechanical loading. 
To conclude, a validated analytical model with a dedicated set of failure criteria, which allows for robust prediction of transverse failure in NCF reinforced composites, is proposed. The model provides a useful tool for accurate and efficient design of NCF composite structures exposed to complex three-dimensional stress states.

\section{Acknowledgement}

The work is mainly funded through Volvo Car Industrial PhD program (VIPP), the Swedish Research Council (VR) 2012-4320, Vinnova 2013-01119 and GKN Aerospace and partly founded via the Vinnova strategic innovation programme, LIGHTer, SRA1.

Robin Olsson at Swerea Sicomp AB are acknowledged for valuable discussions.

\section{References}

[1] Verpoest I, Thanh TC, Lomov S. The TECABS Project: Development of Manufacturing, Simulation and Design Technologies for a Carbon Fiber Composite Car. Proceeding 9th Japan International SAMPE Symposium, 2005, p. 56-61.

[2] Ostergaard MG, Ibbotson AR, Roux OL, Prior AM. Virtual testing of aircraft structures. CEAS Aeronautical Journal 2011:83-103.

[3] Tsai SW, Wu EM. A General Theory of Strength for Anisotropic Materials. Journal of Composite Materials 1971;5:58-80.

[4] Puck A, Schürmann H. Failure analysis of FRP laminates by means of physically based phenomenological models. Composite Science and Technology 2002;62:1633-62.

[5] Pinho S, Darvizeh R. Material and structural response of polymer-matrix fibrereinforced composites. Journal of Composite Materials 2012;46:2313-41.

[6] Marklund E, Asp LE, Olsson R. Transverse strength of unidirectional non-crimp fabric composites: Multiscale modelling. Composite Part B Engineering 2014;65:47-56. 
[7] Camanho PP, Arteiro A, Melro AR, Catalanotti G, Vogler M. Three-dimensional invariant-based failure criteria for fibre-reinforced composites. International Journal of Solids and Structures 2014; Volume 55:92-107.

[8] Lomov S. Non-Crimp Fabric Composites Manufacturing, Properties and Applications. Cambridge: Woodhead Publising Limited; 2011.

[9] Olsson R. Experimental observations on the orthotropic transverse strength of NonCrimp Fabrics composites. Swerea Sicomp TN15006, Piteå, Sweden: 2015.

[10] Drapier S, Wisnomb, Michael R. Finite-element investigation of the compressive strength of noncrimp-fabric-based composites. Composite Science and Technology 1999;59:1287-97.

[11] Edgren F, Asp LE, Joffe R. Failure of NCF composites subjected to combined compression and shear loading. Composite Science and Technology 2006;66:2865-77.

[12] Edgren F, Mattsson D, Asp LE, Varna J. Formation of damage and its effects on noncrimp fabric reinforced composites loaded in tension. Composite Science and Technology 2004;64:675-92.

[13] Kaddour AS, Hinton M. Maturity of 3D failure criteria for fibre-reinforced composites: Comparison between theories and experiments: Part B of WWFE-II. Journal of Composite Materials 2013;47:925-66.

[14] Juhasz J, Rolfes R, Rohwer K. A new strength model for application of a physically based failure criterion to orthogonal 3D fiber reinforced plastics. Composite Science and Technology 2001;61:1821-32.

[15] Asp LE. Local models for NCF composite materials mechanical performance prediction. 16th International Conference on Composite Materials, 2007.

[16] Cuntze RG et al. Neue Bruchkriterien und Festigkeitsnachweise fu“ $r$ unidirektionale Faserkunststoffverbunde unter mehrachsiger Beanspruchung-Modellbildung und Experimente. vol. 5. Düsseldorf: VDI Verlag; 1997.

[17] Marklund E, Asp LE, Varna J. Potential of semi-laminar analyses of NCF composites, Chapter 17 in "Non-crimp Fabric Composites Manufacturing, Properties and Applications", editor Lomov S, Woodhead; 2011, p. 402-38.

[18] Caddell RM, Raghava RS, Atkins AG. Pressure dependent yield criteria for polymers. Materials Science and Engineering 1973;13:113-20.

[19] Bradley WL. Relationship of Matrix Toughness to Interlaminar Fracture Toughness, Chapter 5 in "Application to Fracture Mechanics to Composite Materials", editor Friedrich K. 1989;6:159-87.

[20] Wilhelmsson D, Gutkin R, Edgren F, Asp LE. Numerical analysis of the orthotropic transverse strength of NCF reinforced composites. Texcomp-12 Conference, Raleigh, NC, USA: 2015.

[21] Dassault Systèmes. ABAQUS Documentation. 6.14 ed. Providence, RI, USA: 2014. 


\section{Nomenclature}

$Y_{\mathrm{C}} \quad$ Compressive strength in $Y$.

$Y_{\mathrm{T}} \quad$ Tensile strength in $Y$.

$Z_{\mathrm{C}} \quad$ Compressive strength in $Z$.

$Z_{\mathrm{T}} \quad$ Tensile strength in $Z$.

$S_{\mathrm{L}} \quad$ Longitudinal shear strength.

$E_{11} \quad$ Young's modulus in 1-direction.

$E_{22} \quad$ Young's modulus in 2-direction.

$v_{12} \quad$ Poisson's ratio 1-2.

$v_{23} \quad$ Poisson's ratio 2-3.

$G_{23} \quad$ Shear modulus 2-3.

$\alpha_{11} \quad$ Thermal coefficient for expansion in 1-direction.

$\alpha_{22}$ Thermal coefficient for expansion in 2-direction.

$\alpha_{0} \quad$ Fracture plane for pure transverse compression.

$\alpha \quad$ Fracture plane angle.

$\tau_{\mathrm{T}, \mathrm{MI}} \quad$ Transverse shear on the fracture plane for matrix interface failure in the NCF.

$\tau_{\mathrm{L}, \mathrm{MI}} \quad$ Longitudinal shear on the fracture plane for matrix interface failure in the NCF. 
$\sigma_{\mathrm{N}, \mathrm{MI}}$ Normal stress on the fracture plane for matrix interface failure in the NCF.

$\tau_{\mathrm{T}, \mathrm{B}} \quad$ Transverse shear on the fracture plane for bundle failure in the NCF.

$\tau_{\mathrm{L}, \mathrm{B}} \quad$ Longitudinal shear on the fracture plane for bundle failure in the NCF.

$\sigma_{\mathrm{N}, \mathrm{B}} \quad$ Normal stress on the fracture plane for bundle failure in the NCF.

is In-situ.

$S_{\mathrm{T}} \quad$ Transverse shear strength.

$\theta \quad$ Angle for applied traction, meassured from the 3-direction towards the 2-direction.

$\eta_{\mathrm{T}} \quad$ Transverse friction parameter.

$\eta_{\mathrm{L}} \quad$ Longitudinal friction parameter.

m reference to misaligned coordinate system for fibre kinking.

$X_{\mathrm{T}} \quad$ Tensile strength in $X$.

FI Failure index.

$p \quad$ Parameter for the parabolic criterion.

$Y_{\mathrm{N} \mid \mathrm{T}} \quad$ Calculated tensile strength in the 2-direction for Juhasz' criteria with positive normal stress.

$Y_{\mathrm{N} \mid \mathrm{C}}$ Calculated compressive strength in the 2-direction for Juhasz' criteria with negative positive normal stress. 
$S_{\mathrm{T} \mid \mathrm{T}} \quad$ Calculate transverse shear strength for Juhasz' criteria with positive normal stress

$S_{\mathrm{T} \mid \mathrm{C}} \quad$ Calculate transverse shear strength for Juhasz' criteria with negative positive normal stress.

$\tilde{X}_{\mathrm{C}, \mathrm{T}} \quad$ Basic strength in compression | tension in the 1-direction.

$\tilde{Y}_{\mathrm{C}, \mathrm{T}} \quad$ Basic strength in compression | tension in the 2-direction.

$\tilde{Z}_{\mathrm{C}, \mathrm{T}} \quad$ Basic strength in compression | tension in the 3-direction.

$\tilde{S}_{\mathrm{xy}} \quad$ Basic shear strength in the 1-2-direction.

$\tilde{S}_{\mathrm{yz}} \quad$ Basic shear strength in the 2-3-direction.

$\tilde{S}_{\mathrm{xz}} \quad$ Basic shear strength in the 1-3-direction.

$\sigma_{\mathrm{i}} \quad$ Principle stresses for the Raghava criterion.

$\sigma_{\mathrm{m}} \quad$ Mean stress for the Raghava criterion.

C Compressive yield stresses.

$T \quad$ Tensile yield stresses.

$\mathbf{u}_{\mathbf{i}} \quad$ displacement for location i.

$\bar{\sigma}_{\text {ii }} \quad$ Homogenised stress for $\mathrm{i}=1,2$ and 3. 
$\bar{\tau}_{23} \quad$ Homogenised shear stress.

$m \quad$ Number of rows in failure map.

$n \quad$ Number of columns in failure map.

$Y_{\max } \quad$ Maximum value along $Y$ in failure map.

$Y_{\min } \quad$ Minimum value along $Y$ in failure map.

$Y_{\text {res }} \quad$ Resolution along $Y$ in failure map.

$X_{\max } \quad$ Maximum value along $X$ in failure map.

$X_{\min } \quad$ Minimum value along $X$ in failure map.

$X_{\text {res }} \quad$ Resolution along $X$ in failure map. 